\title{
Core/shell nanomaterials in photovoltaics
}

\author{
Elif Arici, ${ }^{1, \dagger}$ Dieter Meissner, ${ }^{2}$ F. Schäffler, ${ }^{3}$ and N. Serdar Sariciftci ${ }^{1}$ \\ ${ }^{1}$ Linz Institute for organic Solar Cells (LIOS), Physical Chemistry, Johannes Kepler University Linz, \\ Altenberger Str. 69 A4040 Linz, Austria \\ ${ }^{2}$ Fachhochschule Wels, Rosegger Str. 12, A-4600 Wels, Austria \\ 3 Solid State Physics, Johannes Kepler University Linz, Altenberger Str. 69 A4040 Linz, Austria
}

\begin{abstract}
Hybrid materials consist of inorganic nanoparticles embedded in polymer matrices. An advantage of these materials is to combine the unique properties of one or more kinds of inorganic nanoparticles with the film forming properties of polymers. Most of the polymers can be processed from solution at room temperature enabling the manufacturing of large area, flexible and light weight devices. To exploit the full potential for the technological applications of the nanocrystalline materials, it is very important to endow them with good processing attributes. The surface of the inorganic cluster can be modified during the synthesis by organic surfactants. The surfactant can alter the dispersion characteristic of the particles by initiating attractive forces with the polymer chains, in which the particles should be homogenously arranged. In this review, we present wet chemical methods for the synthesis of nanoparticles, which have been used as photovoltaic materials in polymer blends. The photovoltaic performance of various inorganic/organic hybrid solar cells, prepared via spin-coating will be the focus of this contribution.
\end{abstract}

\section{INTRODUCTION}

1.1. Core/shell materials. Nanoclusters of inorganic semiconductor materials, known as nanocrystals have been the subject of intense research in the 1990s. The primary motivation for this increased activity is related to the size dependent new properties of these materials. For example, magnetic and semiconductor nanocrystals exhibit strongly size dependent properties, that may find applications in magnetic storage and microelectronics, key components in information technology [1-3]. The emission and absorption of semiconductor nanocrystals is tuneable by the size as a result of the quantum confinement effect. Also, the use of quantized nanocrystals as fluorescent or absorbing materials in opto-electronic and photovoltaic devices is an interesting and quickly developing area of research [4, 5].

Motivated chemist have spent many years developing synthetic strategies for the preparation of nanocrystalline materials, which should have desirable chemical properties such as high crystallinity, stability against degradation but also flexible handling properties for specific arrangement of the nanocrystals on various substrates. Nowadays, series of nanocrystals with different organic/inorganic shells, shapes and sizes are to find in the literature. Many of them are commercially available.

The very small inorganic crystals are generally unstable due to their high surface tension, and thus have a tendency to transform to larger particles by Ostwald ripening. An important strategy was to create nanoparticles shielded by an organic ligand, the so called "core-shell" particles. The shell can not only avoid the

\footnotetext{
† E-mail: elif.arici@jku.at
}

aggregation and oxidation of the particles, but also can alter the dispersion characteristics of the particles by surface modification, so that the possibility is given to blend the core/shell particles into the polymer matrices, the so called hybrid materials.

An effective strategy of the hybrid solar cell investigations is, to fabricate blends of nanocrystals with semiconductive polymers as a photovoltaic layer. Recently, p-n-junctions by using a p-doping semiconducting polymer (poly-hexylthiophenes) and n-type II-VI semiconductor nanoparticles CdSe have been demonstrated [6]. We will report the studies on CdSe in more detail.

We will further focus on the core/shell systems of the terniary compound semiconductor, CuInSe 2 (and $\mathrm{CuInS}_{2}$ ), which has potential for use in thin-film solar cells because of its high absorption coefficient, band gap, and radiation stability [7-9]. The thin film solar cells based on pure $\mathrm{Cu}(\mathrm{In}, \mathrm{Ga}) \mathrm{Se}_{2}$, investigated by the group of Contreras, have in laboratory conditions up to $18,8 \%$ conversation efficiencies [10]. Investigations on CIS core/shell particles in hybrid solar cells can be a potential alternative for thin-film fabrications.

1.2. Hybrid solar cells. The investigations of inorganic semiconductor nanoparticles embedded into semiconducting polymer blends are promising materials for several reasons:

The classical inorganic semiconductor nanomaterials for photovoltaic applications can have a higher absorption coefficient and photoconductivity than organic nanomaterials.

The $n$ - or p-type doping level of these nanocrystalline semiconductors can easily be varied by synthesis. So that we can study charge transfer in composites 
of n-type and p-type conducting polymers with corresponding inorganic counterparts, respectively. Furthermore, the possibility is given, not only to study electron transfer from p-type polymer to n-type nanoparticle but also hole transfer from the nanoparticle to the polymer after excitation in the nanoparticle.

Typically, inorganic semiconductors in macroscopic dimensions, irrespectively of their size, will absorb all electromagnetic radiation with an energy greater than the band gap. However, if the particles become smaller than the size of the exciton in the bulk semiconductor (typically about $10 \mathrm{~nm}$ ), their electronic structure changes. The electronic properties of such small particles are hence more like those of a giant molecule than an extended solid. The electronic and optical properties of such small particles will depend not only on the material, of which they are composed, but also on their size [11-15]. The lowest energy of optical transition, among others, will increase significantly due to the quantum confinement with decreasing size of the inorganic clusters. For example, the quantized particles of $\mathrm{CuInS}_{2}$ dispersed in acetonitrile can have an energy gap about $2.3 \mathrm{eV}$ in an acetonitrile solution. In comparison to the energy gap of the bulk material, the increase is about $0.9 \mathrm{eV}$ [16].

Band gap tuning in nanoparticles can be used to realization of device architectures, such as tandem solar cells in which the different band gaps can be obtained by modifying only one chemical compound [17, 18]. Since the energy levels of the polymers can be tuned by chemical modification of the backbone-chain and the energy levels of the nanoparticles can be tuned through the size dependent quantum confinement effects, blends of the two materials offer the possibility of tailoring optimal conditions for a solar cell including energy gain from charge transfer for the efficient charge separation and the spectral range of the absorbing light.

The first requirement in inorganic/organic hybrid solar cells is to blend a high concentration of inorganic nanoparticles into the polymer matrix, to form an interpenetrating network (Bulk Heterojunction). At the huge area of $\mathrm{p} / \mathrm{n}$ interfaces, effective charge separation can be realized. The transport of the carriers to the electrodes without recombination is a more complicate problem to solve, since it requires, that once the electrons and holes are separated onto different materials, each carrier type has a pathway to the appropriate electrode without needing to pass through a region of the other material [19]. The nanoscopic morphology of the composite layers play in this manner a fundamental role for efficient solar cell performance. Phase separation in macroscopic scale should be avoided [20].

On the other hand, the performance of nanocrystal based devices is often limited by poor charge transport through the nanoparticle-polymer interface, since the nanocrystals are surrounded by a stabilizing shell of capping molecules. In these cases, it is possible to remove the capping molecules partly by repeated washing with organic solvents providing a better charge carrier exchange between nanocrystals and the conducting polymer.

Another promising technique is the modification of the nanocrystal surface with monomers or oligomers of semiconducting polymers. Subsequent copolymerisation of the nanocrystals with the matrix facilitates incorporation of the inorganic component into the semiconducting polymer and charge transport through the nanocrystal-polymer heterojunction. Details on the methods used to synthesize polymers on the nanoparticle surface can be found elsewhere [2126]. State-of-the art reviews for engineering particle surfaces is also given [27, 28].

In-situ formation of nanoparticles in a high dielectric-constant polymeric medium have been realized for $\mathrm{CuO}$ and $\mathrm{Cu}_{\mathrm{n}} \mathrm{S}$ [29]. To our knowledge, no studies in photovoltaic devices are reported using these methods until yet.

The existence of concentration gradient of the $n$ types nanoparticles within the p-type polymer matrix may allow to optimize the topology of the heterojunction network. Introducing the gradient device morphology leads to increasing the active area for charge separation while decreasing the charge carrier recombination during their transfer to electrodes. To obtain concentration gradient of nanoparticles, the colloid solution have to be placed on top of a polymer film. Diffusion of nanoparticles into the polymer matrix takes place with the penetration depth controlled by temperature, swelling of the polymer layer, and by the size and shape of the nanocrystals. The control over the size and shape of nanoparticles is thereby an additional aspect to outline in the synthesis section below.

Because of the enormous importance of the nature of the shell molecules, the form of the particles and the interactions with the chosen polymer matrix to construct efficient solar cells, special emphasis is given to the synthesis of the "core-shell" particles. An efficient charge transfer from the nanocrystalline inorganic semiconductors to the conduction band of wide-band gap semiconductors (such as $\mathrm{TiO}_{2}, \mathrm{ZnO}$, $\mathrm{Ta}_{2} \mathrm{O}_{5}$ ) in combination with high extinction coefficients of nanocrystals in the visible range makes them very attractive for dye sensitized solar cells. The special case of dye sensitized photo-electrochemical solar cells would go beyond the scope of this review. Recent developments on this systems can be found in [30-37].

\section{SYNTHESIS STRATEGIES OF CORE/SHELL MATERIALS}

There are in principle two distinct synthetic routes to semiconductor nanostructures: physical or chemical procedure. In the "physical" approach, the particles are 


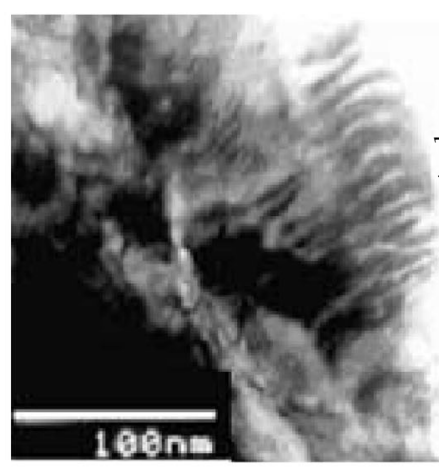

A

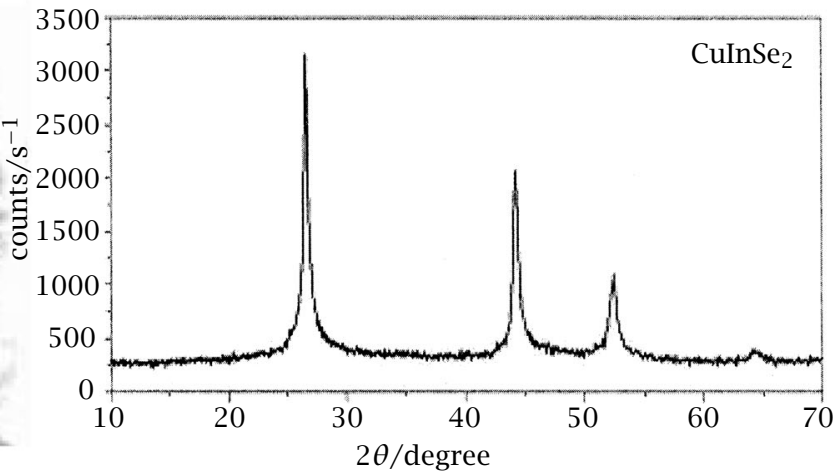

B

Figure 1. (A) TEM images of CISe layers. (B) X-ray diffraction patterns of CISe nanocrystals.

grown by lithographic or molecular beam techniques, whereas in the "chemical" approach, they are formed either by particle growth in various matrices or by the methods of colloidal chemistry.

To give an example, films of $\mathrm{CuInSe}_{2}$ have been grown using such methods as molecular beam epitaxy (MBE) [38-40], liquid-phase epitaxy [41], and halogen vapor phase epitaxy (VPE) [42]. But this methods are quite complicated, so the synthesis of core/shell materials based on an CIS core and organic surfactant as shell can be an interesting alternative on the development of the photovoltaic devices. We will focus, therefore, on the latter approach, which allows us to produce stable particles, which can be handled like ordinary chemical substances.

Core/shell materials have been synthesized based on solvothermal route decomposition of organometallic compounds and colloidal chemistry.

2.1. Solvothermal route. Using the solvothermal approach, the synthesis of III-V semiconductors have been demonstrated, first [43]. An advantage of this method is, that the particles formed are usually crystalline and don't need post-treatment at high temperatures. Also, no organometallic precursor, which can complicate the system and no organic stabilizers are used in this route. The nature of the solvent affects the reaction process and product quality. The synthetic route was extended to the synthesis of nanocrystalline GaN [44] and CuInSe 2 [45].

To synthesize CuInSe $\mathrm{C}_{2}$ (CISe) by the solvothermal route described by Y. Qian et al., a stoichometric mixture of elementary Se, $\mathrm{CuCl}_{2} \cdot 2 \mathrm{H}_{2} \mathrm{O}$ and $\mathrm{InCl}_{3} \cdot \cdot 4 \mathrm{H}_{2} \mathrm{O}$ was loaded into a autoclave, which was than filled with anhydrous ethylenediamine up to $80 \%$ of the total volume. The synthesis was performed at $180^{\circ} \mathrm{C}$ for $15 \mathrm{~h}$. The by-products were removed by washing the product with water and ethanol. The CISe particle were elongated in shape with dimensions of about at least $15 * 60 \mathrm{~nm}$. (Figure 1) [46]. X-ray diffraction pattern of the CISe indicating the chalcopyrite structure is also shown in Figure 1 [50].
Due to its strong basic capacity, an important role of the ethylenediamine is to increase the reactivity of elemental selenium forming $\mathrm{Se}^{2-}$ [45] and to promote the reaction between $\mathrm{InCl}_{3}$ and Se forming the anions $\left(\mathrm{InSe}_{2}\right)^{-}$. Since, ethylenediamine dissolves $\mathrm{CuCl}_{2}$, it can act as a bidentate ligand that could facilitate the formation of chelate complexes of $\left[\mathrm{Cu}(\mathrm{en})_{2}\right]^{+}$and likely forcing the CuInSe $\mathrm{C}_{2}$ grains to grow in one direction. When diethylamine, also a strong base $(\mathrm{pKa}=10.98)$ but without double N-chelation, was used instead of ethylenediamine, spherical CuInSe 2 particles have been obtained [45]. Similar observations between the particle shape and solvent were made also for the synthesis of $\mathrm{PbS}$ nanoclusters [46]. These results indicates that only in a bidentate solvent sustain whisker-like shapes. A major limitation of this method has been that it often leads to aggregated particles embedded in a polymer matrix.

Further wet chemical synthesis methods for nanocrystalline chalcopyrites are given in [48, 49].

2.2. Collodial route. An alternative synthetic approach involves mixing the reagents at a temperature low enough to preclude any appreciable reaction. A controlled dropping of nucleation reagent initiates the reaction. As long as the temperature is adjusted to keep the rate at which the reagents react less or equal to the dropping rate, a controlled reaction conditions are given.

Because the energy of the surface is higher than in the volume, the tendency of the nature is to reduce the surface to volume ratio, which is also the driving force of particle growth in a colloidal route after nucleation. To stabilize the smallest particle in a dispersion, the material exchange between the particles should be hindered. This can be generally realized by adsorbing of charged (electrostatic forces) or bulkier surfactants (greater steric hindrance). Tailoring the ratio of the reagents concentration to that of the surfactants provides a control over the particle size. Higher surfactant to reagent ratios favour the formation of a smaller particle size. Surfactants in solution are only reversibly 


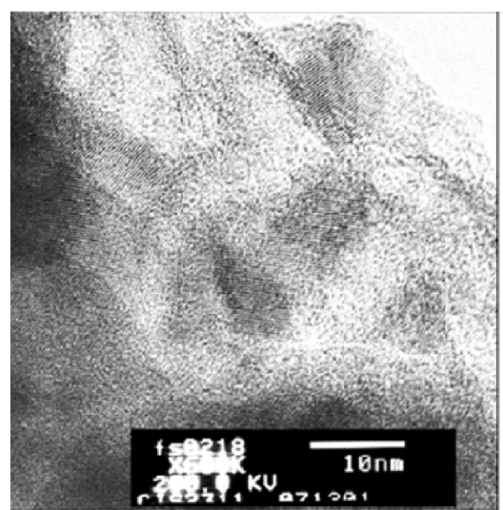

A
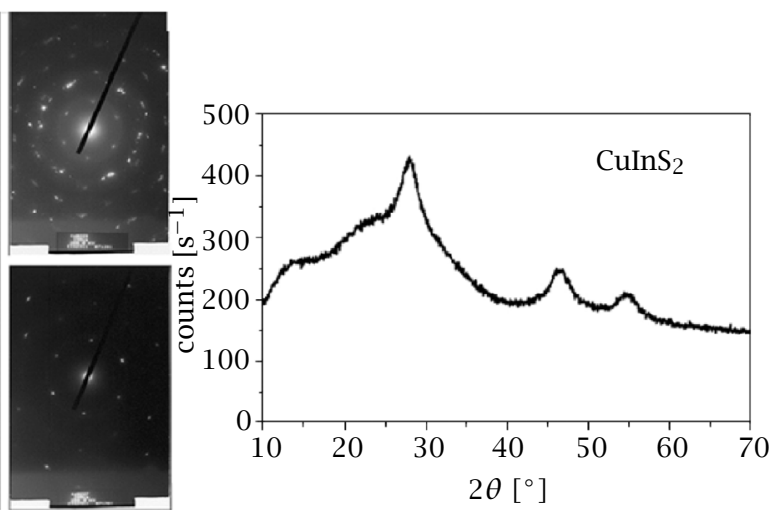

B

Figure 2. (A) HRTEM images of TPP shielded CIS layers. Selected-area electron diffraction pattern of the TPP shielded CIS layers how the presence of polycrystalline nanostructures with the characteristic broad reflections, but also very sharp spots of reflections, indicating the existence of larger particles. (B) X-ray diffraction patterns of CIS nanocrystals.

adsorbed to the surfaces of the reagents and control the particle growth. Also the chemistry of the surfactant plays an important role. For example, bulkier trioctylphosphines provide greater steric hindrance than more compact tributylphosphines, slowing the growing step [51]. The stability of the particle dispersions depends on the temperature and on the solvent. If the interaction between the surfactants and the solvent is favourable, the nanocrystals are then isolated. Introducing an additional solvent, which is miscible with the first solvent but has an unfavourable interaction with the capping group, destabilizes the particle dispersion. A controlled destabilization may lead to form 3D arrays, but a fast growing of the particle cause just their aggregation.

Synthesis of $\mathrm{CuInS}_{2}$ (CIS) nanoparticles was performed by a colloidal route at room temperature [52].

In this synthesis, the used surfactant was triphenyl phosphite (TPP). In the first-step, $\mathrm{InCl}_{3}$ and $\mathrm{CuI}$ was suspended in a mixture of acetonitrile and TPP. The solutions were refluxed in an argon atmosphere to form TPP complexed $\mathrm{InCl}_{3}$ and CuI solutions. Bis(trimethyl silyl)sulfide, which was diluted with TPP, was added for the nucleation, drop by drop to the mixture at room temperature.

A remarkable dynamic colour change of the solution occurs from colourless through yellow to orange and red depending on the amount of added bis(trimethylsilyl) sulfide and on the size of the formed CIS particles. The average particle size increases over the time with a compensating decrease of the nanoparticle number due to the Ostwald ripening. Under cooling conditions, the dispersion is stable for ca. a month.

The HRTEM images of the CIS particles revealed a broad distribution of the particle sizes starting from ten up to tens of nanometers (Figure 2). The shape of the investigated nanograins was mostly sharp-edged, rather than spherical. The electron diffraction pattern displayed in Figure 2 show the presence of polycrystalline nanostructures with the characteristic broad reflection spots, but also very sharp spots of reflections, indicating the existence of larger particles. Both diffraction images have been investigated on the same probe.

In agreement with these results, a pronounced broadening of the CIS diffraction patterns shows the broad size distribution. The three broad reflections at $2 \theta$ values of 27.9 (112), 46,5 (220)/(204) and 55.0 (312)/(116) are those of the chalcopyrite structure of CIS [53]. However, in the range between 15 and $35^{\circ}$ a broad halo appears, probably due to an amorphous contribution. This may indicate that still TPP is present, which may not have been removed completely.

\subsection{Decomposition of organometallic compounds.}

A general scheme for preparing monodisperse nanocrystals via organometallic route requires a single temporally short nucleation event followed by slower growth on the existing nuclei. The highly cited method for making $\mathrm{CdE}(\mathrm{E}=\mathrm{S}$, Se, Te) nanocrystals is that of Murray, Morris and Bawendi [54].

The synthesis of CdSe have been realized by the pyrolysis of the reagents alkyl (dimethylcadmium) and a chalcogen source TOPSe (tri-n-octhylphosphine selenid) in a hot tri-octylphosphine oxide (TOPO) matrix, which is a polar coordinating Lewis base.

The two precursors were dissolved in a liquid phase similar in nature to the matrix. The used liquid phase was tri-octylphosphine (TOP). The nucleation of CdSe nanocrystals was achieved in an inert atmosphere by the sudden introduction of the concentrated reagents in the TOPO matrix. The temperature of the TOPO is sufficient to decompose the reagents $\left(200-350^{\circ} \mathrm{C}\right)$, forming a supersaturation of species in solution that is relieved by nucleation of nanocrystals. Upon nucleation the concentration of these species in solution drops below the critical concentration. Also the temperature 
decreases by adding of the precursors, which have been held by room temperature. Further material can only add to the existing nuclei.

As in the growth of nanocrystals in the matrix, the surfactants dynamically adsorb to the growing crystals allowing the atoms to further adsorbing or deadsorbing, which controls the particle size and shape, while suppressing the aggregation. Each nanoparticle in the sample is surrounded by a TOPO layer [55-57].

It is important to note that the shape of the particles depends strongly on the growth kinetics.

For example, working with highly diluted suspensions lead to small nanocrystals first. Systems with mostly small nanocrystals, on the other hand, exhibit a second growth stage: the high surface energy of small nanocrystals promotes their dissolution at the expense of the larger ones. This growth stage is known as Ostwald ripening. Such slow growth conditions favour the formation of particles with least surface energy. The resulting particle shape is spherical [58]. At higher reagent concentration, relative differences between the growth rates can lead to anisotropic shapes. Controlled grow of crystalline arrays can be further achieved by manipulation of growth kinetics. Combining surfactants, with different strength of the adsorption on the particle surface can be used to vary the growth rates and synthesize anisotropic particle shapes.

Controlled formation of nanoparticles with spherical and rod shapes were realized by CdSe nanocrystals. This is possible because CdSe crystals with the wurtzite structure are intrinstically anisotropic, with a unique c-axis. If the overall growth rate by an extremely high monomer concentration is fast, growth is generally faster along the c-axis. Rods form at high growth rates by unidirectional growth of the c-axis.

The experience showed, that TOPO is not a suitable surfactant for controlled growing rod-like CdSe structures. The resulting rod-like particles are too big in three dimensions and mostly insoluble in common solvents. The blends of this particles in polymers are characterized by large-scale phase separation, which is a disadvantage for photovoltaic applications. Addition of a molecule that coordinates more strongly to cadmium have been used to control the growth rate and shape of the particles. An important discovery was, that hexylphosphonic acid (HPA), which is almost always present as impurities in technical grade TOPO, accentuates the differences in the growth rates among various faces [59]. Controlled formation of CdSe nanocrystals with spherical and rod shapes were realized by the variation of the surfactant ratio of TOPO and HPA. The role of HPA seems to increase the growth rate along the c-axis. At low concentrations of HPA, sphericalshaped nanocrystals form. Intermediate amounts of HPA favour the formation of rods. Higher HPA ratios lead to the formation of arrow shaped nanocrystals. More complex shapes such as teardrop, tetrapod shaped crystals are produced by varying the ratio of surfactants, injection volume and time-dependent monomer concentration [60].

Another strategy to fabricate superstructures from TOPO capped particles with a narrow size distribution is gentle destabilisation of the dispersion. Attractive surface forces of nanoparticles drive self-organization and superlattice formation [61].

The crystallization of TOPO capped CdSe particles, $3.5 \mathrm{~nm}$ in diameter, was carried out in vertically positioned glass tubes. The toluene dispersion of the particles was slowly destabilized by diffusion of methanol, resulting in aggregation of the particles. A buffer layer of propane-2-ol between solvent-non-solvent contact is placed, to decrease the growing rate of the particles. Single crystals grew in the form of perfectly faceted hexagonal plates. The optical behaviour of these three dimensional ordered systems, $100 \mu \mathrm{m}$ in lateral dimensions and $20 \mu \mathrm{m}$ in depth, was very similar to the optical behaviour of individual nanoparticles.

Theoretical aspects for the kinetics of growth of solid state nanocrystals in a liquid medium have been also reported [62].

Nanoparticles of TOPO capped II-VI materials are found to degrade in the presence of light and oxygen, forming unstable chalcogene oxide species [63]. Alternatively, TOPO can be also replaced by other organic groups such as 4-picoline, tris (2-ethylhexyl) phosphate and 4-(trifluoromethyl) thiophenol [64, 65]. Another way to eliminate the degradation is coating the nanoparticle surface by a layer of a higher bandgap semiconductor [66-69]. The relative stability of CdSe (CdS) nanocrystals compared to CdSe is drawn from energetic offsets between the core and shell valence bands. When the CdS shell is present, there is a possibility given, that a hole on the core nanoparticle will recombine with electrons of CdS but not degrade in the presence of oxygen. CdS capped CdSe nanocrystals have been introduced as a new class of electroluminescent materials. The light emitting diodes of ITO/PPV(40 nm)/CdSe (CdS) $(24 \mathrm{~nm}) / \mathrm{Mg} / \mathrm{Ag}$ show low operating voltages, a factor of 8 increase in quantum efficiencies and a factor of 10 increase in lifetimes in comparison to those made by organically capped CdSe nanocrystals [70].

\section{CORE/SHELL MATERIALS IN PHOTOVOLTAIC APPLICATIONS}

Hybrid solar cells can be fabricated via spin-coating from dispersions of core/shell nanocrystals and semiconducting polymers. This technique requires mixing of the components in a good solvent/dispersion, first. The nanocrystals consists of organic shells are readily dispersible in a variety of organic solvents, and they easily form uniform films of closely packed nanocrystals this method. To tailor the right components for an 

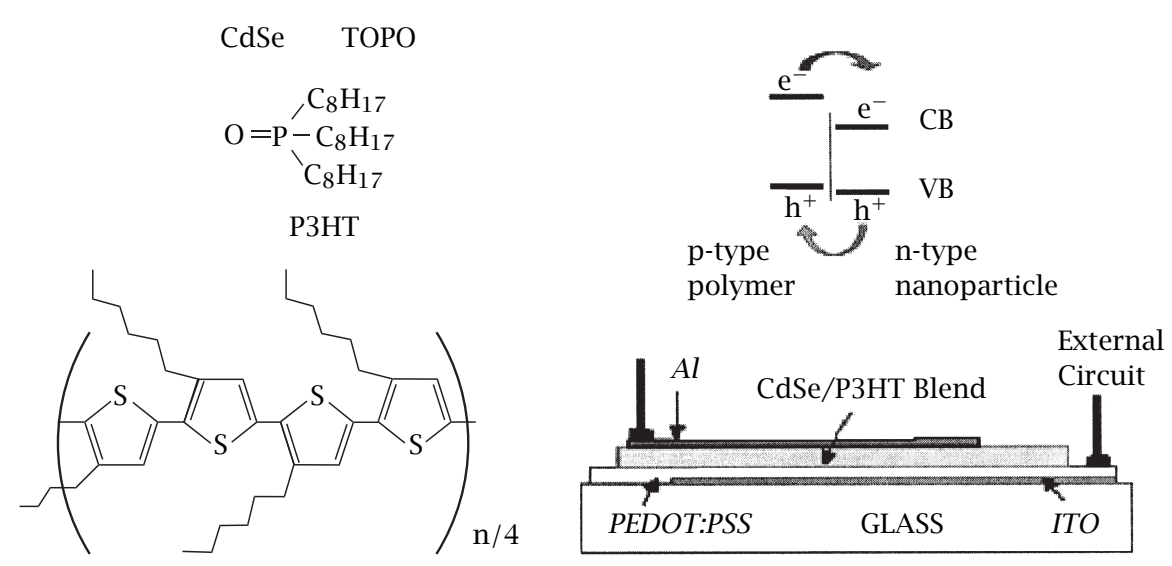

Figure 3. Materials used in CdSe bulkheterojunction solar cells.

ideal morphology of an polymer/inorganic composite film is still a challenge, which will be target of experimental work in future. We will focus on hybrid solar cells based on CdS and CIS nanoparticles.

\subsection{Hybrid solar cells based on CdS. Photo-} voltaic characteristics of CdSe and CdS core/shell particles have been most extensively explored by the group of Alivisatos et al. According to the photoluminescence quenching measurements in composite films of poly(2-methoxy,5-(2'-ethyl-hexyloxy)-pphenylene-vinylene (MEH-PPV) and TOPO capped CdSe nanoparticles $(5 \mathrm{~nm})$, a charge separation at the interface between the polymer and CdSe nanoparticles have been reported [71].

Replacing of the surfactant TOPO by pyridine leads to a more effective charge separation, which have been concluded because of the decrease of MEH-PPV photoluminescence efficiency to a value of ca. 0.01 by mixing CdSe in a composite film. The increase of short circuit photocurrent with increasing amount of CdSe was consistent with this picture. Photoluminescence quenching experiments has been confirmed by the observation of charge on the polymer chain in photo induced absorption measurements [72].

By monochromatic illuminating at $514 \mathrm{~nm}$ of an ITO/CdSe-MEH-PPV/Al cell (95 weight \% CdSe) with a power intensity of $5 \mathrm{~W} / \mathrm{m}^{2}$, they received an open circuit voltage, $\mathrm{V}_{\text {oc }}$ of $500 \mathrm{mV}$ and a short circuit current of about $1 \mu \mathrm{A}$ for an $7.3 \mathrm{~mm}^{2}$ active area. There was a rectification ratio of 100 at $+/-3 \mathrm{~V}$ in the dark and no rectification under illumination. A power conversion efficiency of $0.25 \%$ is obtained. The low quantum efficiency is attributed to the poor transport of charges. TEM images of the films show phase separation for all composite films, unfortunately more pronounced for pyridine capped CdSe particles. Because of the structural traps in the form of incomplete pathways in the percolation network, the separated charges on different materials, recombine with opposite charges before reaching the electrodes. To improve the charge collection, the film morphology should be optimized.

An idea to improve the film morphology was replacing the spherical nanocrystal particles with elongated ones. Van der Waals interactions become stronger as the contact area between two particle increases [73]. Because of this, the tendency for elongated particle to agglomeration will be stronger than spherical particles of the same volume.

The efficient packing of larger nanocrystals may result in a decrease of charge recombination possibilities in a bulk heterojunction hybrid solar cell. Indeed, the use of nanocrystalline rods in conjunction with a regioregular poly(3-hexylthiophene) (P3HT) has resulted in almost an order of magnitude increase in power conversation efficiency, in comparison to the cell discussed above [74]. P3HT is an effective hole transport material in its regioregular form, with field effect hole mobilities up to $0.1 \mathrm{~cm}^{2} \mathrm{~V}^{-1} \mathrm{~s}^{-1}$ [75]. The chemical structures of the materials to construct the CdSe/P3HT and the cell design are shown in Figure 3.

Solar cells were fabricated by spin-casting a solution of 90 weight $\%$ CdSe nanorods $(7 \times 60 \mathrm{~nm})$ in P3HT onto an indium tin oxide glass substrate coated with poly(ethylene dioxythiophene) doped with polystyrene sulfonic acid (PEDOT: PSS) with aluminum as counter electrode. PEDOT:PSS is a highly p-doped polymer, usually coated on the ITO electrode, to smooth the electrode surface and increase the adhesion of organic materials to form good quality layers via spin-coating.

By monochromatic illuminating at $515 \mathrm{~nm}$ of an ITO/PEDOT:PSS/CdSe-P3HT/Al cell with a power intensity of $0.1 \mathrm{~mW} / \mathrm{cm}^{2}$, they received an open circuit voltage, $\mathrm{V}_{\mathrm{Oc}}$ of ca $500 \mathrm{mV}$ and a short circuit current of about $0.02 \mathrm{~mA} / \mathrm{cm}^{2}$. There was a rectification ratio of $10^{5}$ in the dark. A power conversion efficiency of $6.9 \%$ is obtained. Solar cell characteristics of this $7 \mathrm{~nm}$ by $60 \mathrm{~nm}$ nanorod device illuminated with AM 1.5 global light, include a short circuit current of $5.7 \mathrm{~mA} / \mathrm{cm}^{2}$ and an open circuit voltage 

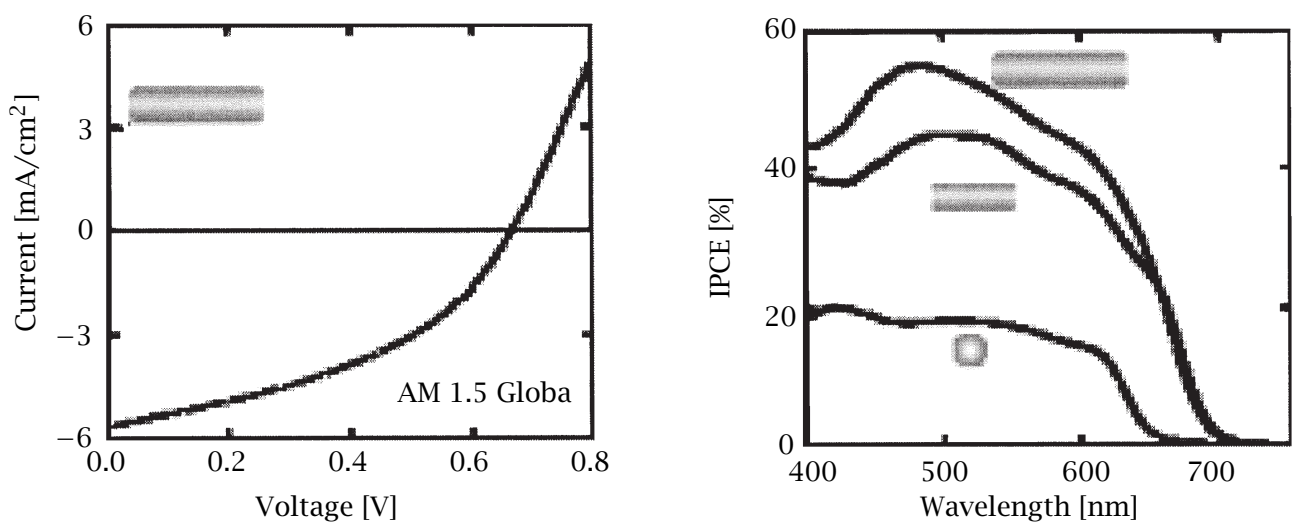

Figure 4. I-V characteristic and IPCE spectra of the CdSe/P3HT solar cells.

of $680 \mathrm{mV}$. The external quantum efficiency (IPCE), which is the percentage of electrons collected per incident photon, increases with increasing length of the nanorods from ca $20 \%$ for $7 \times 7 \mathrm{~nm}$ up to ca $55 \%$ for $7 \times 60 \mathrm{~nm}$ particles. (Figure 4) [6].

3.2. Hybrid solar cells based on CIS. We investigated first blends of poly (2-methoxy-5-(3', $7^{\prime}$-dimethyloctyloxy)-1,4-phenylene vinylene) (MDMO-PPV) and of regioregular poly (3-hexylthiophene) (P3HT) with copper indium diselenide (CISe) and copper indium disulfide (CIS) nanocrystals [46].

The absorption spectrum of films, prepared via spin coating from a diluted acetontirile solutions (0.06 M) of CIS dispersions, exhibits a high shoulder at $700 \mathrm{~nm}$ and a weak absorbance at longer wavelengths. The IPCE on-set is more or less identical with the on-sets of the corresponding absorbance spectra. It is obvious, that quantized CIS particles are photovoltaic active. The fact, that particle growth occurs already by changing the viscosity of the dispersion solution leads us to the assumption, that the organic shell TPP is adsorbed only reversibly on the CIS core. There might be a cooperative interaction between core, shell and solvent. Tailoring the ratio of the concentration of organic shell to that of the right chosen solvent, acetonitrile provides control over the nanoparticle size, since low shell-to-solvent ratio favor the formation of bigger CIS particles. Reducing the amount of acetonitrile leads to weakening of the complexation capability of the TPP and results in a loss of size control. The absorption maximum shifts to the longer wavelengths like the bulk material. The existence of large domains in CIS and MDMO-PPV or P3HT layers, which were spin-coated from a toluene suspension, are in agreement with this argument. In the case of an ITO/PEDOT:PSS/CIS/LiF/Al photovoltaic device, a short circuit density $\mathrm{I}_{\mathrm{sc}}$ of $20 \mu \mathrm{A} / \mathrm{cm}^{2}$ and an open circuit voltage $\mathrm{V}_{\text {oc }}$ of $450 \mathrm{mV}$ have been reported. A fill factor of about 0.25 indicates high serial resistance. However, a low rectification ratio $\mathrm{RR}$ at + and $-2 \mathrm{~V}$ in dark conditions being only 2.5 and under illumination of 6.5 indicates a not optimised diode characteristics. Repeating the measurements and drying the films under argon leads to an improvement of the cell. The chemical structures of materials to optimise the cell performance are given in Figure 5.

Flat interfaces formed in bilayer structures of the CIS and the nicely soluble methanofullerene, 1-(3methoxy carbonyl)-propyl-1-1-phenyl-(6,6) $\mathrm{C}_{61}$ (PCBM) have shown a significant improvement in the photovoltaic performance [76]. PCBM $(\mathrm{HOMO}=-6.4 \mathrm{eV}$, LUMO $=-4.2 \mathrm{eV}$ BISt du sicher mit diesen Angaben?) is the electron transporting and hole blocking "gate"material in this bilayer configuration. An additional advantage of PCBM is its solubility in toluene. TPPcapped CIS particles are also slightly soluble in toluene. However, when PCBM is deposited onto a previously prepared CIS film, some swelling of the CIS layer may occur some roughening of the formed interfaces between two layers.

The CIS/PCBM double layer exhibits a reasonable diode behavior with a typical current versus voltage I-V response. Illuminating the cell with $80 \mathrm{~mW} / \mathrm{cm}^{2}$ white light from a solar simulator produces an open circuit voltage, $\mathrm{V}_{\text {oc }}$ of $710-790 \mathrm{mV}$ and a short circuit current density of ca. $0.26 \mathrm{~mA} / \mathrm{cm}^{2}$. The calculated fill factor of the diode is 0.44 . Conversion efficiency of $0.086 \%$ is obtained. The rectification ratio low at $+/-2 \mathrm{~V}$ is 2,5 in the dark and 3.5 under illumination.

It is obvious, that layers of TPP capped CIS, contrary to the layers consisting TOPO capped CdS nanocrystals, a photovoltaic response can be detected without removing the organic ligand. TPP seems to be weakly bound on the inorganic core and not totally insulating the particles from each other.

We investigated a bulk heterojunction device prepared from CIS and highly p-doped poly(3,4-ethylenedioxythiophene): poly(4-styrenesulfonate) complex (PEDOT:PSS). The main point thereby was to prepare a well-percolating film, while at the same time to avoid the CIS particle- growth during the preparation [77]. The advantage of PEDOT-PSS is in this aspect its 


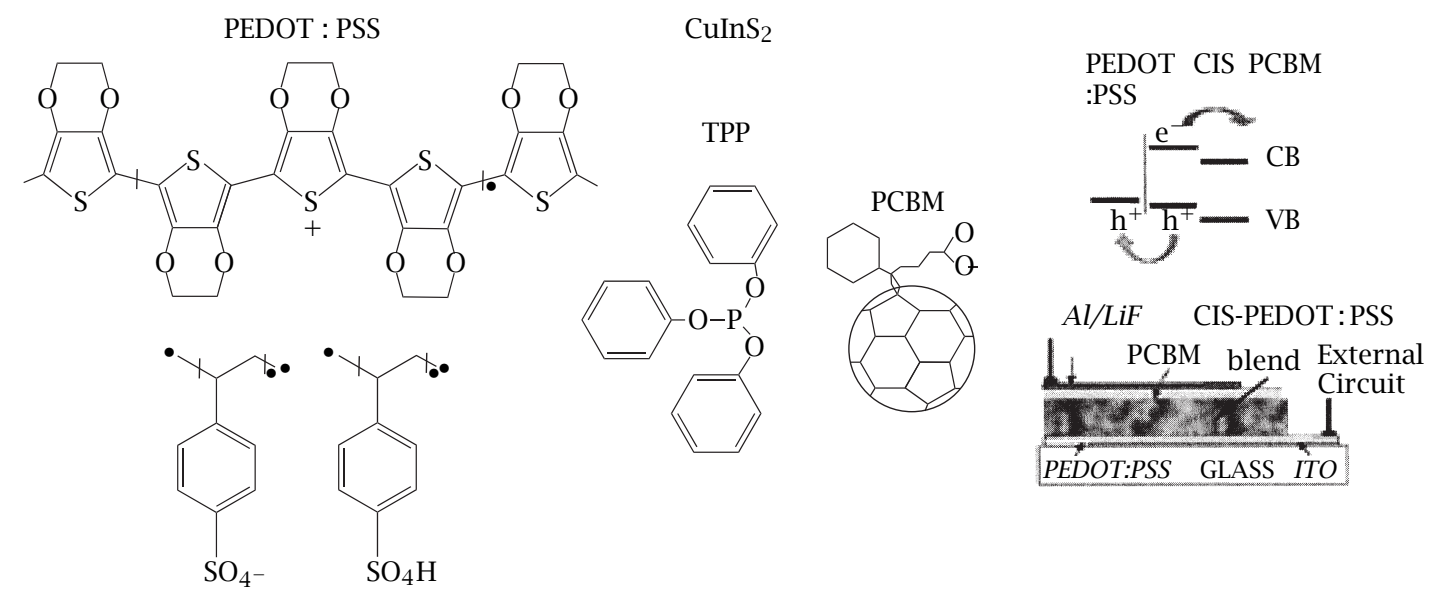

Figure 5. Materials used in CIS hybrid solar cells and schematic structure of bulk heterojunction devices ITO/CISPEDOT:PSS/PCBM/AI.

solubility in acetonitrile/TPP mixtures. PEDOT:PSS does not absorb in the visible range. Thereby it is easy to investigate the size distribution of the CIS particles by studying the absorbance behavior of CIS depending on the preparation. The aim was to form an interconnected network of the nanoparticle and of the polymer, so that the charge separation can occur at the very large interfacial area of the nanoparticle-polymer contacts. The optical and microscopic investigations on the layers of CIS-PEDOT:PSS blends showed, that the nanoparticles do not grow during spin-coating from an acetonitrile dispersion of CIS and PEDOT:PSS.

In our systems, due to the high HOMO level of PEDOT : PSS ( $\mathrm{E}_{\mathrm{HOMO}} \sim-5.0 \mathrm{eV}$ ) not far from the work function of the $\mathrm{Al}$ electrode $(\sim-4.3 \mathrm{eV})$ an ohmic contact can be formed even to this electrode short circuiting the cell.

By white light illuminating of an ITO/CIS-PEDOT: $\mathrm{PSS} / \mathrm{LiF} / \mathrm{Al}$ cell with a power intensity of $80 \mathrm{~mW} / \mathrm{cm}^{2}$, we received an open circuit voltage, $\mathrm{V}_{\mathrm{Oc}}$ of $150 \mathrm{mV}$ and a short circuit current density of $4 \mu \mathrm{A} / \mathrm{cm}^{2}$. The calculated fill factor of the diode is 0.1 . A conversion efficiency of only $0.003 \%$ is obtained. There is no rectification in the dark and a rectification ratio of $2.2 \mathrm{at}+1-2 \mathrm{~V}$ under illumination [77].

In order to prevent the short circuiting contact between the hole conductor PEDOT:PSS and the electron collecting Al-electrode a PCBM interlayer was introduced. Thereby a white light illumination of the cell with a power intensity of $80 \mathrm{~mW} / \mathrm{cm}^{2}$, produced an open circuit voltage, $\mathrm{V}_{\text {oc }}$ of $220 \mathrm{mV}$ and a short circuit current density of $0.84 \mathrm{~mA} / \mathrm{cm}^{2}$. The rectification ratio in the dark is 13.1 at $+/-2 \mathrm{~V}$ and 11.3 under illumination. The cell configuration is shown in Figure 5. The holes move along the PEDOT:PSS network towards the high work function electrode ITO and the electrons, in turn can move trough the PCBM layer, where they can easily be transported to $\mathrm{Al}$ electrode whereas the holes

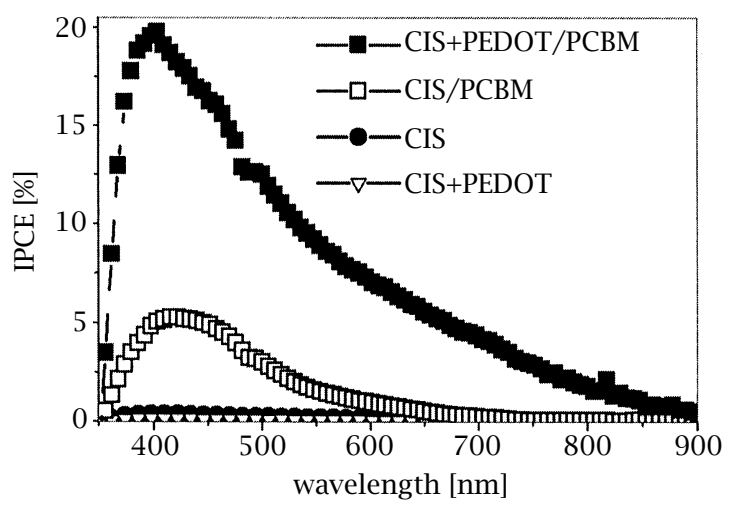

Figure 6. IPCE spectra of CIS photovoltaic devices using different device structures. (Reprinted from Ref. 132, Arici et al., Adv. Funct. Mater., 13 (2), (2003) in print. With permission from Advanced Functional Materials.)

are blocked by the high ionization energy of the PCBM HOMO level.

The most significant differences between the different cell types investigated can be seen from the incident photon to current conversion efficiencies (IPCE) spectra (Figure 6). The IPCE on-set are more or less identical with the on-sets of the corresponding absorbance spectra. The photocurrent response is not limited by the absorption depth of the film. The obtained photocurrent for the CIS single-layer is very poor, the photogenerated excitons mostly recombine, before they can reach the electrodes. However, current generation can significantly be improved by adding PCBM as a strong electron acceptor.

Because of the ohmic contact between PEDOT: PSS and the Al electrode, the IPCE value is decreased for CISPEDOT:PSS mono layers as compared to the mono layer of nanocrystalline CIS. The IPCE value of CIS-PEDOT: PSS/PCBM bilayer gives by far the best results. 


\section{OUTLOOK}

A new field of scientific research has grown out of the new strategy of creating composite layers of organic/inorganic blends with huge surface areas for charge separation. More recent approaches, particularly those based on core-shell particles, have been shown to be effective in producing composite blends by easy processing techniques like spin-coating. The synthetic approaches outlined provide a high level of flexibility, thus allowing the cell-design to be variable. On the other hand, the morphology of organic/inorganic blends is still not well-controlled.

Because of the enormous importance of the nature of the shell molecules, the form of the particles and the interactions with the chosen polymer matrix to construct efficient solar cells, future research to a large extent, continue to be focused on optimising existing approaches, as well as developing new procedures, in order to modify the surface properties of nanoparticles. Self-assembling of nanoparticles into colloidal crystals are expected to be an additional strategy for the morphology control.

An interesting extension of the core/shell nanoparticles may be the manufacturing of semi-conductive polymer layers on the surfaces of nanoparticles instead of using insulating organic shells between them. Finally, a significant goal is the development of the long-term stability.

\section{ACKNOWLEDGEMENT}

Financial support by the Bundesministerium für Bildung und Forschung in Germany, No: 01SF0026, is gratefully acknowledged. We express our sincere thanks to Dr. Malik, Inorganic Chemistry, Manchester University, England.

\section{REFERENCES}

[1] M. H. Huang, S. Mao, H. Feick, H. Yan, Y. Wu, H. Kind, E. Weber, R. Russo, and P. Yang, Science 292 (2001), 1897.

[2] S. Sun, C. B. Murray, D. Weller, L. Folks, and A. Moser, Science 287 (2000), 1989.

[3] S. A. Empodocles and M. G. Bawendi, Science 278 (1997), 2114.

[4] M. Gao, C. Lesser, S. Kirstein, H. Möhwald, A. L. Rogach, and H. Weller, J. Appl. Phys. 87 (2000), 2297.

[5] M. Gao, B. Richter, S. Kirstein, and H. Möhwald, J. Phys. Chem. B 102 (1998), 4096.

[6] W. U. Huynh, W. W. Dittmer, and A. P. Alivisatos, Science 295 (2002), 2425.

[7] V. Nadenau, D. Braunger, D. Hariskos, M. Kaiser, C. Koble, M. Ruckh, R. Schaffer, D. Schmid, T. Walter, S. Zwergart, and H. W. Schock, Prog. Photogr. Res. Appl. 3 (1995), 363.
[8] L. Stolt, M. Bodegard, J. Kessler, M. Ruckh, K. O. Velthaus, and H. W. Schock, Proc. 11th Euro. Photovoltaic Solar Energy Conf., Monteux, Harwood Academic, Chur, Switzerland, 1993.

[9] S. Niki, P. J. Fons, A. Yamada, O. Hellman, T. Kurafuji, S. Chichibu, and H. Nakanishi, Appl. Phys. Lett. 69 (1996), 647.

[10] M. A. Contreras, et al., Prog. Photovoltaics 7 (1999), 311.

[11] H. Weller, Angew. Chem. Int. Ed. Engl. 32 (1993), 41.

[12] M. L. Steigerwald and L. E. Brus, Acc. Chem. Res. 23 (1990), 283.

[13] A. P. Alivisatos, Science 271 (1996), 933.

[14] S. A. Empedocles and M. G. Bawendi, Acc. Chem. Res. 32 (1999), 389.

[15] C. J. Murphy and J. L. Coffer, Appl. Spectr. 56 (2002), 16.

[16] E. Arici, N. S. Sariciftci, and D. Meissner, Molecular Crystals Liquid Crystals 383 (2002), 129.

[17] M. Green, Physica E: Low Dimensional Systems and Nanostructures (Amsterdam, Netherlands) 14 (2002), 65.

[18] M. Green, Progress in Photovoltaics 9 (2001), 123.

[19] C. Brabec, N. S. Sariciftci, and J. C. Hummelen, Adv. Func. Mat. 11 (2001), 15.

[20] S. Shaheen, C. J. Brabec, F. Padinger, T. Frommherz, J. C. Hummelen, and N. S. Sariciftci, Appl. Phys. Lett. 78 (2001), 841.

[21] H. T. Oyama, R. Sprycha, Y. Xie, R. E. Partch, and E. Matijevic, J. Colloid Interface Sci. 160 (1993), 298.

[22] S. M. Marinakos, D. A. Schultz, and D. L. Feldheim, Adv. Mater. 34 (1999), 11.

[23] S. M. Marinakos, J. P. Novak, L. C. Brousseau, A. B. House, E. M. Edeki, J. C. Feldhaus, and D. L. Feldheim, J. Am. Chem. Soc. 121 (1999), 8518.

[24] Y. Yang, J. Huang, S. Liu, and J. Shen, J. Mater. Chem. 7 (1997), 131.

[25] S. W. Haggata, D. J. Cole-Hamilton, and J. R. Fryer, J. Mater. Chem. 7 (1997), 1969.

[26] F. M. Pavel and R. A. Mackay, Langmuir 16 (2000), 8568.

[27] F. Caruso, Adv. Mater. 13 (2001), 11.

[28] K. Yoshinaga, Surfactant Science Series 92 (2000), 626.

[29] A. Weitz, J. Worrall, and F. Wudl, Adv. Mater. 12 (2000), 106.

[30] A. F. Nogueira, J. R. Durrant, and M. A. De Paoli, Adv. Mater. 13 (2001), 826.

[31] A. F. Nogueira and M. A. De Paoli, Sol. Energy Mater. Sol. Cells 61 (2000), 135.

[32] G. Katsaros, T. Stergiopoulos, I. M. Arabatzis, K. G. Papadokostaki, and P. Falaras, J. Photochem. Photobiology A: Chemistry 149 (2002), 191.

[33] K. Murakoshi, R. Kogure, Y. Wada, and S. Yanagida, Chem. Lett. 5 (1997), 471. 
[34] Y. Saito, T. Kitamura, Y. Wada, and S. Yanagida, Synth. Met. 131 (2002), 185.

[35] T. J. Savenije, J. M. Warman, and A. Goossens, Chem. Phys. Lett. 287 (1998), 148.

[36] D. Gebeyehu, C. J. Brabec, and N. S. Sariciftci, Thin Solid Films 403-404 (2002), 271.

[37] D. Gebeyehu, C. J. Brabec, N. S. Sariciftci, D. Vangeneugden, R. Kiebooms, D. Vanderzande, F. Kienberger, and H. Schindler, Synth. Met. 125 (2002) 279.

[38] B. Schumann, T. Tempel, and G. Kuhn, Sol. Cells 16 (1986), 43.

[39] A. N. Tiwari, S. Blunier, K. Kessler, V. Zelezny, and H. Zogg, Appl. Phys. Lett. 65 (1994), 2299.

[40] A. N. Tiwari, S. Blunier, M. Filmoser, H. Zogg, D. Schmid, and H. W. Schock, Appl. Phys. Lett. 65 (1994), 3347.

[41] H. Takenoshita, Sol. Cells 16 (1986), 65.

[42] O. Igarashi, J. Cryst. Growth 130 (1993), 343.

[43] Y. Qian, Adv. Mater. 11 (1999), 1101.

[44] Y. Xie, Y. Qian, W. Wang, S. Zhang, and Y. Zhang., Science 272 (1996), 1926.

[45] B. Li, Y. Xie, J. Huang, and Y. Qian, Adv. Mater. 11 (1999), 1456.

[46] E. Arici, H. Hoppe, A. Reuning, N. S. Sariciftci, and D. Meissner, 17th European Photovoltaic Solar Energy Conference, Proceedings of the International Conference held in Munich, Germany, 2001.

[47] W. Wang, Y. Geng, Y. Qian, M. Ji, and X. Liu, Adv. Mater. 10 (1998), 1479.

[48] J. Xiao, Y. Xie, R. Tang, and Y. Qian, J. Solid State Chem. 161 (2001), 179.

[49] A. B. Mandale, S. D. Sathaye, and K. R. Patil, Mater. Lett. 55 (2002), 30.

[50] H. Hwang, et al., Thin Solid Films 67 (1980), 83.

[51] C. T. Black, C. B. Murray, R. L. Sandstrom, and S. Sun, Science 290 (2000), 1131.

[52] C. Czekelius, M. Hilgendorff, L. Spanhel, I. Bedja, M. Lench, G. Müller, U. Bloeck, D. Su, and M. Giersig, Adv. Mater. 11 (1999), 643.

[53] D. Suri, K. Nagpal, and G. Chadha, J. Appl. Crystallogr. 22 (1989), 578.

[54] C. B. Murray, D. J. Norris, and M. G. Bawendi, J. Am. Chem. Soc. 115 (1993), 8706.

[55] T. Trindade and P. O’Brien, Adv. Mater. 8 (1996), 61.

[56] T. Trindade, P. O’Brien, and X. Zhang, Chem. Mater. 9 (1997), 523.
[57] J. E. Katari, V. L. Colvin, and A. P. Alivisatos, J. Phys. Chem. 98 (1994), 4109.

[58] X. G. Peng, J. Wickham, and A. P. Alivisatos, J. Am. Chem. Soc. 120 (1998), 5343.

[59] X. G. Peng, L. Manna, W. D. Yang, J. Wickham, E. Scher, A. Kadavanich, and A. P. Alivisatos, Nature 404 (2000), 59.

[60] L. Manna, E. Scher, A. Kadavanich, and A. P. Alivisatos, J. Am. Chem. Soc. 122 (2000), 12700.

[61] D. V. Talapin, E. V. Shevchenko, A. Kornowski, N. Gaponik, M. Haase, A. L. Rogach, and H. Weller, Adv. Mater. 13 (2001), 1868.

[62] C. D. Dushkin, S. Saita, K. Yoshie, and Y. Yamaguchi, Adv. Col. Int. Sci. 88 (2000), 37.

[63] J. E. Bowen-Katari, V. L. Colvin, and A. P. Alivisatos, J. Phys. Chem. 98 (1994), 4109.

[64] K. Yoshinaga, Surfactant Science Series 92 (2000), 626.

[65] M. Kuno, J. K. Lee, B. O. Dabbousi, F. V. Mikulec, and M. C. Bawendi, J. Chem. Phys. 106 (1997), 9869.

[66] X. Peng, M. C. Schlamp, A. V. Kadavanich, and A. P. Alivisatos, J. Am. Chem. Soc. 119 (1997), 7019.

[67] Y. Cao and U. Banin, J. Am. Chem. Soc. 122 (2000), 9692.

[68] Y. Cao and U. Banin, Angew. Chem. Int. Ed. 38 (1999), 3692.

[69] M. T. Harrison, S. V. Kershaw, A. L. Rogach, A. Kornowski, A. Eychmüller, and H. Weller, Adv. Mater. 12 (2000), 123.

[70] M. C. Schlamp, X. Peng, and A. P. Alivisatos, J. Appl. Phys. 82 (1997), 5837.

[71] N. C. Greenham, X. Peng, and A. P. Alivisatos, Phys. Rev. B 54 (1996), 17628.

[72] D. S. Ginger and N. C. Greenham, Synth. Met. 101 (1999), 425.

[73] M. R. Böhmer, J. Colloid. Interface Sci. 197 (1998), 251.

[74] U. Wendy, X. P. Huynh, and A. P. Alivisatos, Adv. Mater. 11 (1999), 923.

[75] H. Sirringhaus, N. Tessler, and R. H. Friend, Science 280 (1998), 1741.

[76] E. Arici, N. S. Sariciftci, and D. Meissner, Molecular Crystals Liquid Crystals 383 (2002), 129.

[77] E. Arici, N. S. Sariciftci, and D. Meissner, Adv. Funct. Mater. 13 (2003), 1. 


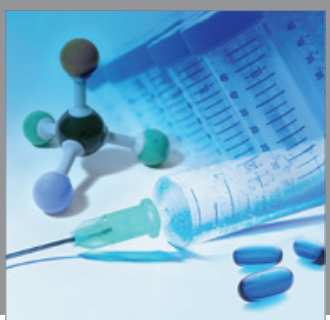

International Journal of

Medicinal Chemistry

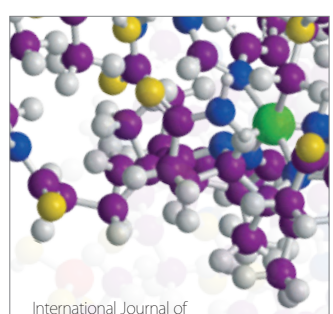

Carbohydrate Chemistry

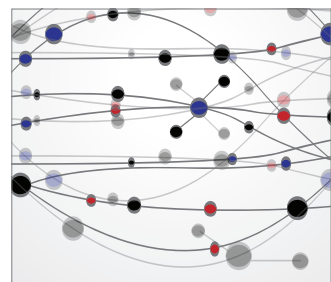

The Scientific World Journal
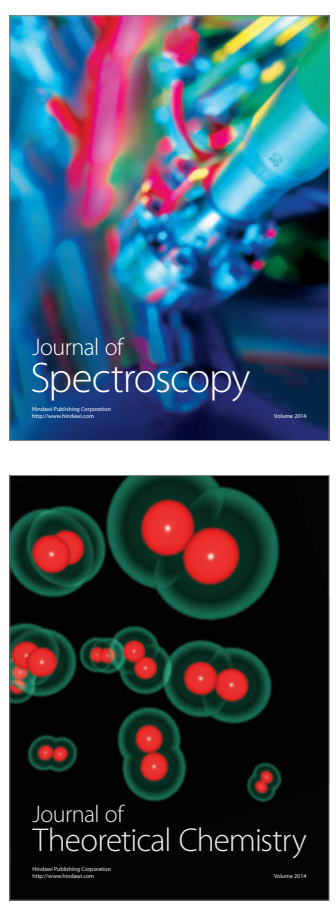
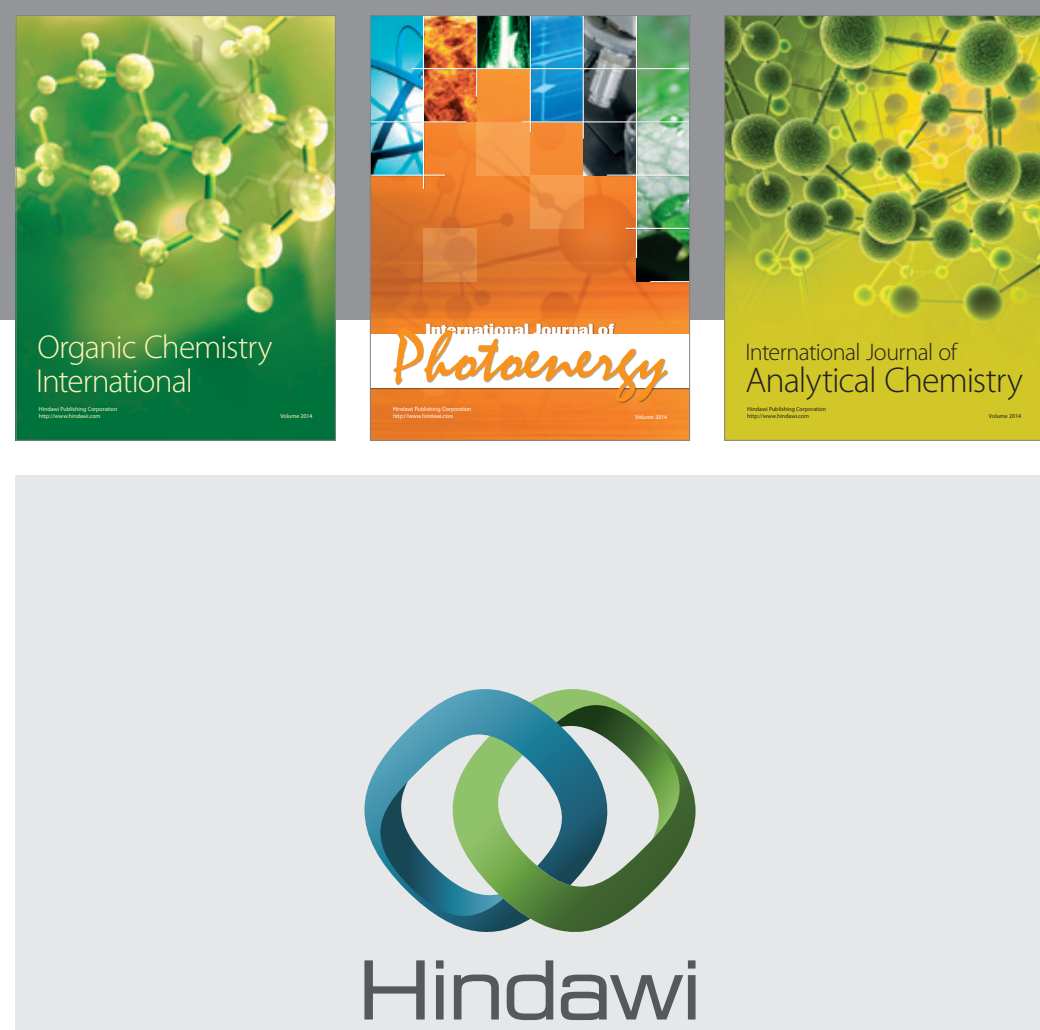

Submit your manuscripts at

http://www.hindawi.com
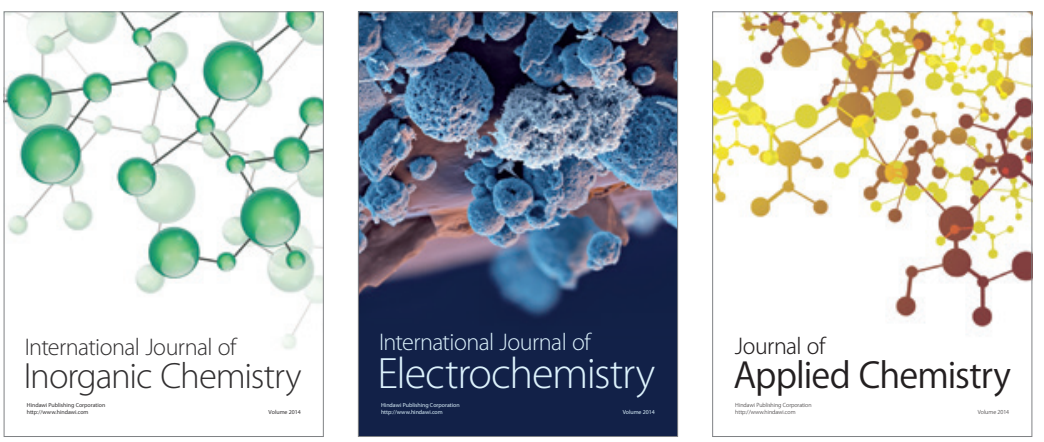

Journal of

Applied Chemistry
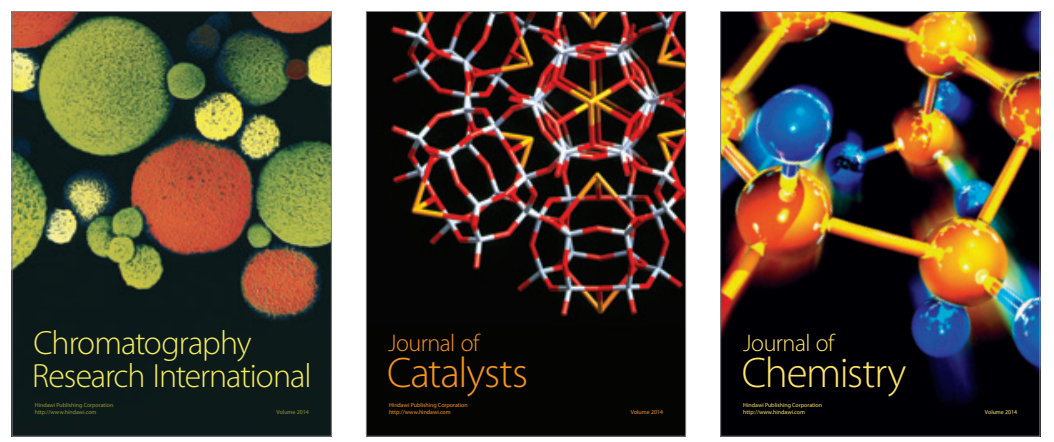
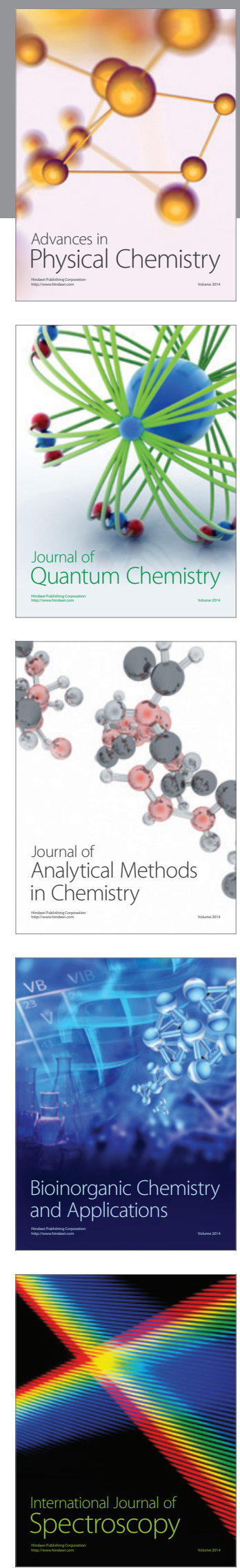